\title{
Conceptual Design Optimization of an Augmented Stability Aircraft Incorporating Dynamic Response and Actuator Constraints
}

\author{
Jason Welstead* \\ NASA Langley Research Center, Hampton, VA, 23681, United States of America \\ and Gilbert L. Crouse, Jr. ${ }^{\dagger}$ \\ Sierra Nevada Corp., Louisville, CO, 80027, United States of America
}

\begin{abstract}
Empirical sizing guidelines such as tail volume coefficients have long been used in the early aircraft design phases for sizing stabilizers, resulting in conservatively stable aircraft. While successful, this results in increased empty weight, reduced performance, and greater procurement and operational cost relative to an aircraft with optimally sized surfaces. Including flight dynamics in the conceptual design process allows the design to move away from empirical methods while implementing modern control techniques. A challenge of flight dynamics and control is the numerous design variables, which are changing fluidly throughout the conceptual design process, required to evaluate the system response to some disturbance. This research focuses on addressing that challenge not by implementing higher order tools, such as computational fluid dynamics, but instead by linking the lower order tools typically used within the conceptual design process so each discipline feeds into the other. In this research, flight dynamics and control was incorporated into the conceptual design process along with the traditional disciplines of vehicle sizing, weight estimation, aerodynamics, and performance. For the controller, a linear quadratic regulator structure with constant gains has been specified to reduce the user input. Coupling all the disciplines in the conceptual design phase allows the aircraft designer to explore larger design spaces where stabilizers are sized according to dynamic response constraints rather than historical static margin and volume coefficient guidelines.
\end{abstract}

\section{Nomenclature}

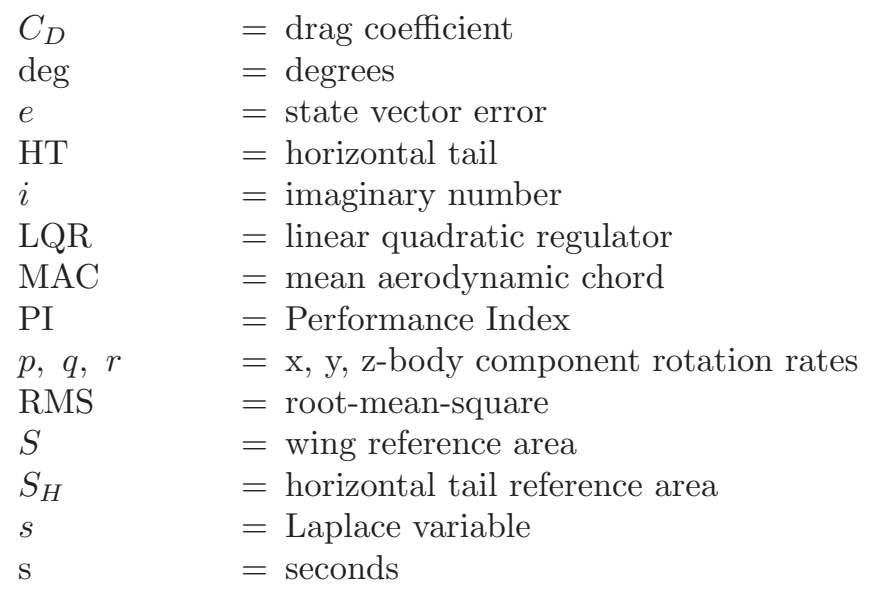

*Aerospace Engineer, NASA Langley Research Center 1 N Dryden St. M/S 442, Hampton, VA 23681, Member AIAA

${ }^{\dagger}$ Formerly Associate Professor, Department of Aerospace Engineering, 211 Davis Hall, Auburn University, AL 36849, Associate Fellow AIAA 


\begin{tabular}{|c|c|}
\hline $\mathrm{T}$ & $=$ matrix transpose \\
\hline $\bar{U}$ & $=$ steady-state velocity in body-fixed stability axis \\
\hline$u$ & $=$ control input vector \\
\hline$u, v, w$ & $=\mathrm{x}, \mathrm{y}, \mathrm{z}$-body components of velocity \\
\hline VT & $=$ vertical tail \\
\hline$x$ & $=$ state vector \\
\hline & $=$ performance output vector \\
\hline & $=$ control surface deflection angle \\
\hline & $=$ root-mean-square deviation in continuous turbulence \\
\hline & $=$ time constant \\
\hline$\theta, \theta, \psi$ & $=$ roll, pitch, and yaw Euler angles \\
\hline
\end{tabular}

\section{Introduction}

S stated by Roskam, "Rather significant improvements in airplane payload-range performance are possible A by reducing tail areas and relaxing the requirement for inherent static and dynamic stability." ${ }^{1}$ It is well known that decreasing the static stability of an airplane will result in overall performance increases. Relaxed static stability (RSS), the intentional reduction of static margin, has been used in modern military aircraft along with commercial transport aircraft such as the McDonald Douglas MD-11. ${ }^{2}$ However, even though the benefits are well known, stability and control is rarely applied in the conceptual design process. As the demand to reduce fuel burn, emissions, and noise increases to meet the NASA Fixed Wing Project next generation aircraft goals, it becomes imperative to incorporate more disciplines into the conceptual design process beyond the traditional sizing, weight estimation, aerodynamics, and performance.

For subsonic flight, the total aircraft parasite drag consists of mostly skin-friction and pressure drag. ${ }^{3}$ With fairings and surface blending, much of the pressure drag can be reduced leaving skin-friction drag as the largest contributor to parasite drag. Effectively, if the wetted area of the aircraft can be minimized, the skin-friction drag will be minimized resulting in decreased parasite drag. Numerous designs have reduced this wetted area by using tailless configurations such as the Convair F-106, Convair B-58, Messerschmitt 163B, and the Northrop Grumman B-2. ${ }^{4,5}$ All of these configurations have low aspect ratios and thus increased induced drag. It would be beneficial if both the induced and parasite drag could be reduced simultaneously.

A statically stable, conventional configuration requires a download on the horizontal tail for trim, increasing the required main wing lift coefficient resulting in greater induced drag. On the other hand, positive lift produced by the horizontal tail also results in greater than minimal induced drag due to the horizontal stabilizer span being less than the main wing. Any lift from the stabilizer results in a total induced drag penalty and a reduction in the total aerodynamic efficiency of the aircraft. For a conventional configuration, eliminating lift produced by the empennage minimizes aircraft total induced drag. Reducing the aircraft static margin is a way to reduce the induced drag on the empennage but requires some form of stability augmentation. ${ }^{6-8}$ According to Raymer, "[A] modern and sophisticated aft-tail aircraft is designed to a slight level of instability so that it normally flies with an upload, not a download on its tail. This is the very reason that computerized flight control systems with artificial stability were developed and put into production." 3

Classical aircraft design uses volume coefficients when sizing an aircraft's empennage, which tends to produce conservative estimates for the stabilizer areas. ${ }^{9}$ As a result, the surface area for the horizontal and vertical stabilizers exceed the necessary area for adequate dynamic stability. Additionally, this fails to take into account the benefits of augmented stability provided by the active control systems in modern aircraft. As mentioned previously, a large majority of parasite drag results from skin friction over the wetted area of the aircraft. Augmenting the aircraft's stability through active control will allow the stabilizers to be reduced in size thus reducing the total wetted area of the aircraft. In doing so, not only will the induced drag be reduced from the reduced tail load but the total parasite drag will be reduced from the decrease in wetted area.

Conceptual design typically focuses on the interaction between the disciplines of aerodynamics, sizing, weight estimation, and performance. ${ }^{10}$ Any inclusion of control surface design during conceptual design is often limited to estimating control surface sizes from historical data while assuming control effectiveness is proportional the force and the moment arm. ${ }^{9,11}$ Often times, flight dynamics and control (FDC) and handling qualities are examined after the aircraft geometry and structural properties have been defined, 
which inevitably leads to sub-optimal designs or even configurations that have deficient flying qualities. FDC is incredibly important when it comes to the overall safety and certification of an aircraft, ${ }^{9,12}$ and deficient handling qualities lead to reduced aircraft performance, large cost increases, and delays as the configuration must be re-evaluated or redesigned.

Collaboration between traditional conceptual design disciplines and flight dynamics and controls is essential when designing for relaxed static stability (RSS). By designing for RSS, aircraft performance is increased through reduction of wetted area drag, trim drag, and total weight. ${ }^{9,11,13}$ For a transport aircraft of conventional stability margins, the horizontal tail accounts for $20-30 \%$ of the aircraft-lifting surface and approximately $2 \%$ of the aircraft empty weight. ${ }^{11}$ Any reduction in size of the horizontal stabilizer from RSS will provide a significant benefit in reduced drag and aircraft gross weight. However, relaxation of stability margin gives a detrimental effect to the aircraft's handling qualities that must be compensated or augmented. ${ }^{11}$ It is this correlation between performance gains using RSS and degraded handling qualities that make it essential to incorporate flight dynamics and control into the conceptual design phase, especially when using a multidisciplinary design optimizer.

Perez, Liu, and Behdinan in Refs. 9-11 incorporate FDC into a multidisciplinary optimization (MDO) including handling qualities, as specified in MIL-F-8785C, on a Boeing 737 class aircraft. The optimizer recognized the benefit of RSS by reducing the static margin, moving the wing apex location, shifting the CG location, and reducing the horizontal stabilizer (down 28\%) and control surface areas. By integrating active control into the optimization loop, adequate handling qualities were ensured with feedback control to augment the stability. Additionally, reduced control deflections were necessary for trim decreasing trim drag. Perez et al. used the decoupled equations of motion with a short period approximation and single-input single-output (SISO) to control the dutch roll and short period modes by selecting appropriate gains.

Morris et al. uses the method of linear matrix inequalities (LMI) to place constraints on the maximum actuator deflection, actuator rate, and pole placement limitations. ${ }^{14}$ This method relies heavily upon the work of Boyd ${ }^{15}$ and Kaminer ${ }^{16}$ to place constraints on the static feedback gain matrix, $K$, to obtain desired handling qualities. Morris expands his work in Ref. 17 by translating the MIL-STD-1797A guidelines into state variance constraints to be used in the development of a state feedback control law using optimal control.

References 18-21 describe the SimSAC project using the CEASIOM software which takes a different approach than the methods described by Perez et al. and Morris et al. The SimSAC project uses higher order tools, such as computational fluid dynamics (CFD), to iterate a conceptual design. The project has had good success showing the benefits of relaxed static stability, but the higher order tools reduce the ability to explore a large design space with numerous varying geometric parameters. The optimization time using CEASIOM is on the order of weeks instead of the much faster methods described by Perez and Morris and the methods presented in this work.

The focus of this research is to provide a performance gain by reducing the total aircraft drag and weight by allowing relaxed static stability and incorporating flight dynamics and control into the conceptual design process. By reducing aircraft drag, the overall range and endurance of the aircraft will increase resulting in greater operational capability and reduced fuel burn.

This paper discusses the development of several key components in the MDO framework including flight dynamics and control, stability augmentation, atmospheric disturbances, and coupling with a vortex lattice aerodynamic code. Fully coupled equations of motion were developed and implemented into a stability augmentation system (SAS) that utilizes a linear quadratic regulator where a focus has been placed on reducing the user input. Athena Vortex Lattice (AVL) ${ }^{22}$ was used to calculate the stability derivatives of a Cessna 182 like model to be used in the coupled equations of motion. Unlike much of the work previously discussed, state variable transient response to perturbations and atmospheric disturbances were used as a measure of handling qualities instead of the more traditional MIL-F-8785C or MIL-STD-1797B military specifications. As the configurations and control systems become more complex, the traditional decoupled longitudinal and lateral modes may no longer be present, which poses a problem when using pole placement techniques. This research eliminates the necessity of identifying the dynamics modes and measuring the handling qualities in a qualitative manner and quantitatively evaluates the transient response according to the SAE-AS94900 ${ }^{23}$ specification. Results from varying the horizontal and vertical tail volume coefficients are presented. 


\section{Methodology}

\section{A. System Dynamics}

The fully coupled equations of motion were derived and linearized about a steady state condition resulting in a state-space representation of the perturbation equations. State feedback was chosen for the controller structure due to the guaranteed closed-loop stability properties of the controller which work well in a diverse design space. Although it is rare to have all the states available, the focus of this research was not to design a robust controller but rather to incorporate active control into the conceptual design space that would give a stable solution.

The perturbation equations were derived assuming a symmetric geometry with zero steady sideslip. The steady-state thrust terms were solved for explicitly and substituted into the state-space form of the perturbation equations. The implicit, state-space form of the fully coupled perturbation equations is given by

$$
E \dot{x}=\hat{A} x+\left[\begin{array}{c:c}
\hat{B} & \hat{B}_{g}
\end{array}\right]\left\{\begin{array}{c}
\delta \\
\hdashline u_{g}
\end{array}\right\}
$$

where $\hat{B}_{g}$ is the gust input matrix. The state and control vectors are

$$
\begin{aligned}
& x=\left[\begin{array}{lllllllll}
u & v & w & p & q & r & \phi & \theta & \psi
\end{array}\right]^{\mathrm{T}} \\
& \delta=\left[\begin{array}{lll}
\delta_{e} & \delta_{a} & \delta_{r}
\end{array}\right]^{\mathrm{T}} \\
& u_{g}=\left[\begin{array}{lll}
u_{\text {gust }} & v_{\text {gust }} & w_{\text {gust }}
\end{array}\right]^{\mathrm{T}}
\end{aligned}
$$

with the full definition of the matrices in Eq. 1 given in the Appendix. Inverting the generalized inertial matrix, $E$, one obtains the standard, explicit, state-space model

$$
\dot{x}=E^{-1} \hat{A}+E^{-1}\left[\begin{array}{l:l}
\hat{B} & \hat{B}_{g}
\end{array}\right]\left\{\begin{array}{c}
\delta \\
\hdashline u_{g}
\end{array}\right\}=A x+\left[\begin{array}{l:l}
B & B_{g}
\end{array}\right]\left\{\begin{array}{c}
\delta \\
\hdashline u_{g}
\end{array}\right\}
$$

A linear actuator model was added to include the actuator dynamics in the system dynamics. The actuator transfer function for each control surface was modeled by a simple-lag filter given by

$$
\frac{\delta(s)}{u(s)}=\frac{1}{\tau s+1}
$$

where $\tau$ is the time constant of the filter. The time constant was chosen to be the same for all three actuators and was selected as $\tau=1 /(20.2)$ s. ${ }^{6}$ The perturbation equations described by Eqs. 1-3 were augmented to include the actuator dynamics while neglecting all unsteady terms, thrust terms (except $C_{T_{x_{u}}}$ ), steady-state roll $(\bar{R})$, and negligibly small terms. AVL was used for the aerodynamic model which does not include thrust in the analysis and therefore those terms had to be neglected. The velocity dependent thrust term, $C_{T_{x_{u}}}$, was included in the perturbation equations as it would be the largest in magnitude for the configuration studied in this research. It was approximated using Ref. 24 to be

$$
C_{T_{x_{u}}}=-3\left(\bar{C}_{D}-\bar{C}_{L} \sin \bar{\theta}\right)
$$

The augmented perturbation equations are presented in compact form in Eq. 6 with the matrix definitions given in the Appendix. Again, the hat used in the Appendix equations indicates that a matrix has not be premultiplied by the inverted generalized inertial matrix, $E_{a u g}$.

$$
\left\{\begin{array}{c}
x \\
\delta
\end{array}\right\}=A_{\text {aug }}\left\{\begin{array}{c}
x \\
\hdashline \delta
\end{array}\right\}+\left[B_{u}: B_{g_{a u g}}\right]\left\{\begin{array}{c}
u \\
\hdashline u_{g}
\end{array}\right\}
$$

The actuator input vector, $u$, is defined as

$$
u=\left[\begin{array}{lll}
u_{e} & u_{a} & u_{r}
\end{array}\right]^{\mathrm{T}}
$$




\section{B. Optimal Control by Linear Quadratic Regulator}

The complete dynamic system of an aircraft incorporating active control is complex requiring numerous loop closures to provide adequate closed-loop system response. Classical control relies on the iterative selection of gains to achieve the closed-loop system stability, but there is no guarantee the gains chosen will be optimum. Modern control theory takes advantage of current computing power where numerous linear equations can be solved simultaneously to obtain a set of gains that minimizes a chosen performance index (PI). It is in the selection of the performance index that the true engineering of the controller occurs.

A linear quadratic regulator (LQR) can be used to simultaneously close all the loops in a linear, timeinvariant (LTI), multi-input multi-output (MIMO) system. With the closure of all the loops, the gains are solved simultaneously negating the need for successive loop closure as required in classical control theory. Extremely versatile, the LQR is capable of using performance indices with state and control weighting, time weighting, and derivative weighting of the state in both state feedback and output feedback control structures. Without any restrictions on the gains, other than closed-loop stability is required, the LQR may choose to zero a gain, thus leaving a loop unclosed. Additionally, a compensator may be used in the form of filters, integral and derivative controllers. This flexibility makes it an excellent tool for finding optimal gains for a controller in an LTI system.

As a regulator, any non-zero states are driven to zero in such a way that a chosen performance index is minimized. By driving all the states to zero the system is returned to the steady-state condition with the least amount of cost, thus being an optimal solution. This is ideal for a stability augmentation system where any deviation from the steady state is undesired.

A set of nonlinear equations of motion can be linearized about a steady-state condition and represented in state-space form as given by

$$
\dot{x}=A x+B u
$$

where both $x$ and $u$ are functions of time. The state vector is a vector of perturbations from the steady state condition that the regulator drives to zero. With the state-feedback control law,

$$
u=-K x
$$

the closed loop system takes the form

$$
\dot{x}=(A-B K) x \equiv A_{c} x
$$

A performance output can be defined as ${ }^{6}$

$$
z=H x
$$

where $z$ is a combination of states. For a regulator, $z$ can be set to the error of a specific state such as a non-zero pitch rate that should be driven to zero.

The LQR finds the optimal gains through the minimization of a performance index that integrates the values of both the state and control vectors over time. The impact of each state and control is weighted in the performance index through the use of weighting matrices. The standard performance index for the LQR is $^{6}$

$$
J=\frac{1}{2} \int_{0}^{\infty}\left(x^{\mathrm{T}} Q x+u^{\mathrm{T}} R u\right) d t
$$

with $Q \geq 0, R>0$. The performance output, $z$, can be incorporated into the performance index such that $Q=H^{\mathrm{T}} H$.

A limitation of the linear quadratic method is the $n \times \mathrm{x} n$ entities that must be chosen in the weighting matrix $Q$ where the values may not correspond to a performance objective due to an observability requirement, initially presented by Kalman ${ }^{25}$ and discussed by Stevens and Lewis. ${ }^{6}$ This results in a trial-and-error method of selection for $Q$ where the entries are varied until an acceptable transient response is obtained. This method of design is highly undesirable.

Eliminating the restriction of the observability requirement in the selection of $Q$ allows for the entries to be chosen strictly on desired performance objectives. With specified performance objectives, the structure of the PI and the number of entities to be chosen for the weighting matrices can be reduced and the closed-loop response is dependent on the design of the performance index.

A strength of the linear quadratic method is the flexibility in the selection of the performance index structure. The standard PI given in Eq. 12 can be modified by adding time and derivative weighting of the 
states. A benefit of the time-weighted performance index is that it can satisfy the observability requirement freeing the selection of the weighting matrices. The standard PI only lightly penalizes small errors due to a slow pole(s) with small residue resulting in the time to reach a steady state condition to be rather large. The time-weighted performance index heavily penalizes errors that occur late in the response and as a result suppresses the effect of a slow pole and lightly damped settling behavior. ${ }^{6}$

The derivative of the state can sometimes be a more accurate representation of the workload on a control system and should be weighted in the performance index rather than the state itself. For example, the rate of change of the elevator is a more accurate representation of required actuator power than the deflection angle itself.

The standard PI for the linear quadratic regulator was modified to include the state error and derivative weighting of the states given by

$$
J=\frac{1}{2} \int_{0}^{\infty}\left(t^{k} e^{\mathrm{T}} e+u^{\mathrm{T}} R u+\dot{z}^{\mathrm{T}} W \dot{z}\right) d t
$$

where $R$ has the same definition as in the standard performance index, $Q$ has been set equal to zero, $W$ is a new weighting matrix on the states' rate of change. The state error, $e$, is defined as

$$
e=z \equiv H x
$$

where $P=H^{\mathrm{T}} H$.

The solution to Eq. 13 can be shown to be

$$
J=\frac{1}{2} \operatorname{tr}\left(P_{k} X\right)
$$

subject to the nested Lyapunov equations given by

$$
\begin{aligned}
& 0=g_{0} \equiv A_{c}^{\mathrm{T}} P_{0}+P_{0} A_{c}+P \\
& 0=g_{1} \equiv A_{c}^{\mathrm{T}} P_{1}+P_{1} A_{c}+P_{0} \\
& \vdots \\
& 0=g_{k-1} \equiv A_{c}^{\mathrm{T}} P_{k-1}+P_{k-1} A_{c}+P_{k-2} \\
& 0=g_{k} \equiv A_{c}^{\mathrm{T}} P_{k}+P_{k} A_{c}+k ! P_{k-1}+K^{\mathrm{T}} R K+A_{c}^{\mathrm{T}} H^{\mathrm{T}} W H A_{c}
\end{aligned}
$$

with $X$ defined as

$$
X=x(0) x^{\mathrm{T}}(0)=H^{-1} z(0) z^{\mathrm{T}}(0)\left(H^{-1}\right)^{\mathrm{T}}
$$

The solution to this minimization problem is dependent upon the initial conditions, $x(0)$. A simple solution for eliminating the dependence on the initial state is to average the performance of a set of linearly independent initial conditions. This is equivalent to a random variable uniformly distributed on the surface of a unit sphere of dimension equal to the length of the state vector. ${ }^{26}$ In essence, instead of minimizing the performance index in Eq. 15, the expected value of $\mathrm{J}, E\{J\}$, is minimized such that

$$
E\{J\}=\frac{1}{2} E\left\{x^{\mathrm{T}}(0) P x(0)\right\}=\frac{1}{2} \operatorname{tr}(P X)
$$

where the symmetric $n \times n$ matrix

$$
X \equiv E\left\{x(0) x^{\mathrm{T}}(0)\right\}
$$

is the initial autocorrelation of the state. For the regulator problem, it is practical to set $X=I$ since it is desired to drive arbitrary nonzero states to zero. ${ }^{6}$

A emphasis was placed on reducing the number of entities that must be chosen in the weighting matrices. As a regulator with state feedback, any nonzero state was considered an error. The performance output, $z$, was selected as

$$
z=e=\left[\begin{array}{llllllllllll}
u & \beta & \alpha & p & q & r & \phi & \theta & \psi & \delta_{e} & \delta_{a} & \delta_{r}
\end{array}\right]^{\mathrm{T}}
$$

with the performance output matrix $H$ defined as

$$
H=\frac{180}{\pi} \operatorname{diag}\left\{\begin{array}{llllllllllll}
\frac{\pi}{180} & \frac{1}{U} & \frac{1}{U} & 1 & 1 & 1 & 1 & 1 & 1 & 1 & 1 & 1
\end{array}\right\}
$$


The performance output was chosen to give one degree of error equal weighting as one foot per second in the performance index. This was chosen to maintain the generality of the controller while reducing the number of entities to be chosen in the performance index.

The derivative-weighting matrix $W$ was chosen as

$$
W=\operatorname{diag}\left\{\begin{array}{llllllllllll}
0 & 0 & 0 & 0 & 0 & 0 & 0 & 0 & 0 & 1 & 1 & 1
\end{array}\right\}
$$

to place a small penalty on high control surface deflection rates.

The controls-weighting matrix $R$ was selected as

$$
R=0.1\left[\begin{array}{lll}
1 & 0 & 0 \\
0 & 1 & 0 \\
0 & 0 & 1
\end{array}\right]
$$

where smaller values of $R$ gives greater authority to the control inputs. However, values less than those given in Eq. 23 produce transient responses negligibly different than results using Eq. 23.

\section{Atmospheric Disturbances}

To stress the control system, atmospheric disturbances were modeled and used to test the response of the system to deviations from the steady-state condition. Including these disturbances adds validity to the model and eliminates unrealistic configurations. Two atmospheric disturbance models were used as suggested in military standard MIL-STD$1797 \mathrm{~A}:{ }^{27}$ a continuous turbulence model in the frequency domain and a discrete gust model in the time domain.

\section{Continuous Turbulence}

The von Kármán continuous turbulence model was used as specified in MIL-STD-1797A. The spectra are of the form

$$
\begin{aligned}
& \phi_{u_{g}}(\Omega)=\sigma_{u}^{2} \frac{2 L_{u}}{\pi} \frac{1}{\left[1+\left(1.339 L_{u} \Omega\right)^{2}\right]^{5 / 6}} \\
& \phi_{v_{g}}(\Omega)=\sigma_{v}^{2} \frac{L_{v}}{\pi} \frac{1+\frac{8}{3}\left(1.339 L_{v} \Omega\right)^{2}}{\left[1+\left(1.339 L_{v} \Omega\right)^{2}\right]^{11 / 6}} \\
& \phi_{w_{g}}(\Omega)=\sigma_{w}^{2} \frac{L_{w}}{\pi} \frac{1+\frac{8}{3}\left(1.339 L_{w} \Omega\right)^{2}}{\left[1+\left(1.339 L_{w} \Omega\right)^{2}\right]^{11 / 6}}
\end{aligned}
$$

Table 1. RMS gust intensities, fps.

\begin{tabular}{cccc}
\hline \hline Altitude (ft) & Light & Moderate & Severe \\
\hline 500 & 6.6 & 8.6 & 15.6 \\
1,750 & 6.9 & 9.6 & 17.6 \\
3,750 & 7.4 & 10.6 & 23.0 \\
7,500 & 6.7 & 10.1 & 23.6 \\
15,000 & 4.6 & 8.0 & 22.1 \\
25,000 & 2.7 & 6.6 & 20.0 \\
35,000 & 0.4 & 5.0 & 16.0 \\
45,000 & 0 & 4.2 & 15.1 \\
55,000 & 0 & 2.7 & 12.1 \\
65,000 & 0 & 0 & 7.9 \\
75,000 & 0 & 0 & 6.2 \\
85,000 & 0 & 0 & 5.1 \\
\hline \hline
\end{tabular}

where $L_{n}$ is the length scale and $\sigma_{n}$ is the root-mean-square intensity of the continuous turbulence. The spatial frequency, $\Omega$, used in the von Kármán form of the spectra is related to the temporal frequency, $\omega$, by $\Omega=\omega / \bar{U}$. The spectra are converted to functions of $\omega$ using the simple relationship

$$
\phi_{n_{g}}(\omega)=\frac{1}{\bar{U}} \phi_{g}(\Omega), \quad n=u, v, w
$$

The mean square of the output can be found be integrating the square of the system transfer function $i \omega$ times the power spectral density of the continuous response for all frequencies as shown in Eq. 26.

$$
\sigma_{n}^{2}=2 \int_{0}^{\infty}|G(i \omega)|^{2} \phi_{n_{g}}(\omega) d \omega, \quad n=u, v, w
$$

For isotropic atmospheric turbulence, a requirement of both the von Kármán and Dryden forms, the turbulence length scales and mean-square intensities are the same for the three velocity components and is shown 
by

$$
\begin{gathered}
\sigma_{u}^{2}=\sigma_{v}^{2}=\sigma_{w}^{2} \\
L_{u}=L_{v}=L_{w}
\end{gathered}
$$

For clean air turbulence above 2,500 feet, the length scale of the turbulence is 2,500 feet in the von Kármán form and 1,750 feet for the Dryden form which is used in the discrete gust model described in the next section.

The RMS intensities to be used in Eq. 24 were specified in Table 4 in Section 3.2.1.5.1 of SAE-AS94900. ${ }^{23}$ The table gives the RMS intensities for numerous altitudes with varying probability of exceedance. The intensities corresponding to light, moderate, and severe with probabilities of exceedance of $10^{-2}, 10^{-3}$, and $10^{-5}$ are given in Table 1. Altitudes used between data points were linearly interpolated to allow for any desired altitude.

\section{Discrete Gust}

The discrete gust model has the "1-cosine" profile defined by Eq. 28 and illustrated in Fig. 1

$$
\begin{array}{rrrl}
v & =0, & x & <0 \\
v & =\frac{V_{m}}{2}\left(1-\cos \frac{\pi x}{d_{m}}\right), & 0 \leq x & \leq d_{m} \\
v & =0, & x & >d_{m}
\end{array}
$$

where $V_{m}$ is the magnitude of the gust and $d_{m}$ is one-half the total gust length. The gust length was chosen to provide the maximum system excitation by tuning the gust to the system natural frequencies. ${ }^{23}$ The magnitude of the gust was chosen according to MIL-STD-1797A using Fig. 2.

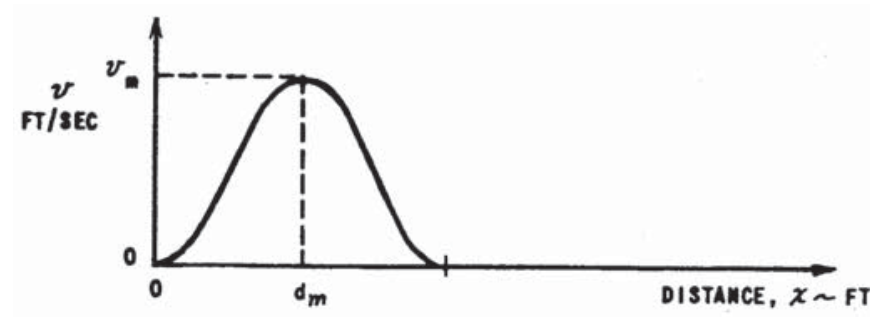

Figure 1. "1-cos" discrete gust profile. ${ }^{28}$

The Dryden turbulence scales, and the rootmean-square intensities from Table 1, are used in Fig. 2. SAE-A94900 specifies the maximum discrete gust to be a single full wave with a peak amplitude of 60 fps. Figure 2 is for light to moderate turbulence where the probability of exceedance is $10^{-2}$. The user of the tool has the option to use either the calculated gust magnitude taken from Fig. 2, or use the severe turbulence case of $60 \mathrm{fps}$.

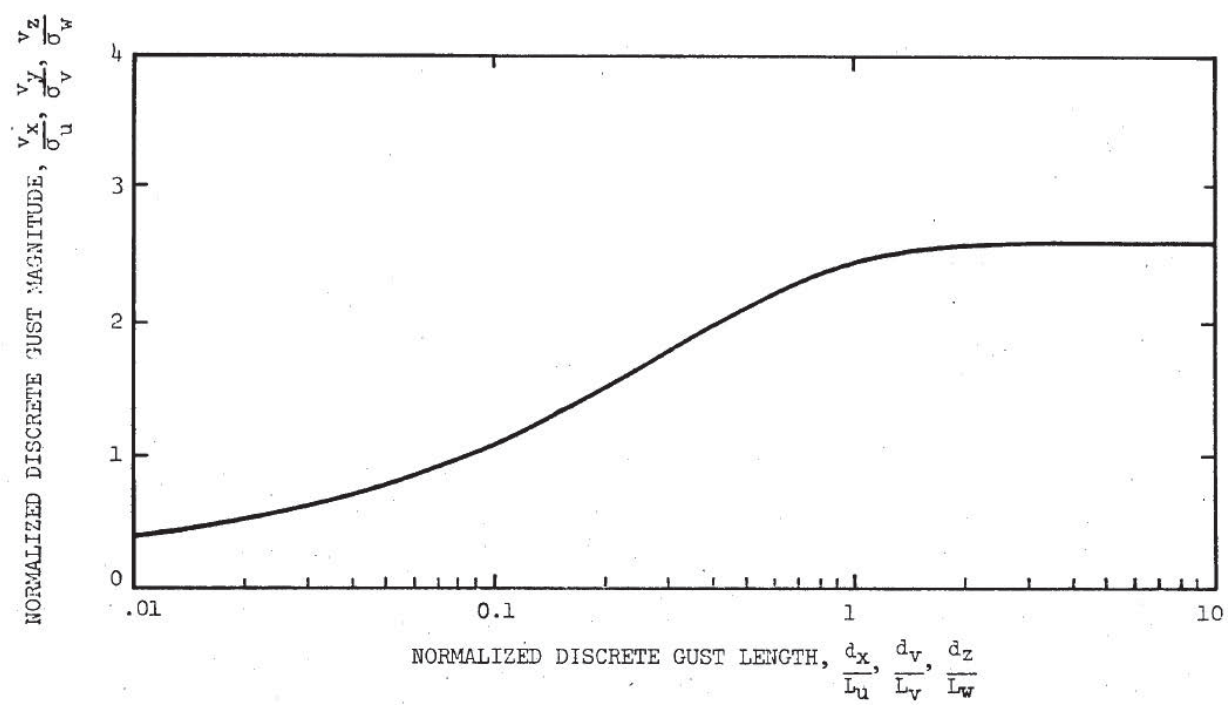

Figure 2. Normalized discrete gust for determining gust magnitude. ${ }^{27,29}$ 


\section{Dynamic Performance Constraints}

Reducing horizontal and vertical stabilizer areas results in degraded flying qualities of any configuration. Damping of the dynamic modes is directly affected by those stabilizer areas. The ability to trim any moments also reduces with the empennage size due to reduced control effector area. Flying quality enhancements must be provided through active control systems that reduce or eliminate undesired residual oscillations. The control system workload increases with decreasing empennage area resulting in a point where the control system cannot provide adequate flying qualities.

A 20 degree maximum control deflection angle was chosen to limit the authority of the control system. Twenty degrees was chosen to allow a margin of safety in addition to avoiding the nonlinear effects of large control surface deflections. The total deflection angle was calculated as the sum of the steady-state deflection and any perturbation deflections. This was checked during the discrete gust simulation discussed previously.

Military specification SAE-AS94900 ${ }^{23}$ specifies performance requirements on the transient response of the attitude angles that allows for quantitative evaluation of the flying qualities without having to identify specific dynamic modes; this is advantageous because the traditional dynamics modes may not even exist due to the complex dynamics provided by active control. Assessing the transient response allows for the flying qualities to be evaluated for any geometric configuration with any active control system.

As specified in SAE-AS94900, the root-mean-square deviations in pitch attitude must be less than or equal to five degrees in a continuous turbulence field. The control system must also be capable of returning the pitch attitude to within plus or minus 0.5 degrees of the steady-state condition, resulting from a five degree perturbation in pitch, within five seconds for aircraft in classes I-III, defined in MIL-STD-1797A. The roll attitude requirements are similar to the pitch attitude requirements with the exception of a ten degree root-mean-square deviation in roll requirement in continuous turbulence, and a static accuracy of one degrees within five seconds from a roll perturbation. In continuous turbulence, the heading angle, $\psi$, must have a root-mean-square heading deviation of less than or equal to five degrees. ${ }^{23}$

While climbing up to a maximum rate of 2000 feet per minute, the control system must be capable of leveling off and achieving a static airspeed accuracy of plus or minus 10 knots or $2 \%$ of the reference airspeed, whichever is greater. This accuracy must be achieved within 30 seconds of engaging the airspeed hold. Any residual oscillations within the static accuracy margin must have a period of oscillation greater than 20 seconds. This requirement was modeled as a small perturbation in the pitch angle, $\theta$.

Table 2. Summary of dynamic performance constraints.

\begin{tabular}{lcr}
\hline \hline Description & Test Condition & Constraint \\
\hline 20 deg max control deflection & pert. and gust & never exceed \\
5 deg pitch & perturbation & $\pm 0.5 \mathrm{deg}$ in $<5 \mathrm{~s}$ \\
5 deg roll & perturbation & $\pm 1.0 \mathrm{deg}$ in $<5 \mathrm{~s}$ \\
airspeed hold & perturbation & $\pm 10 \mathrm{kts}$ or $2 \%<30 \mathrm{~s}$ \\
pitch deviation & cont. turbulence & $\sigma_{\theta}<5 \mathrm{deg}$ \\
roll deviation & cont. turbulence & $\sigma_{\phi}<10 \mathrm{deg}$ \\
heading deviation & cont. turbulence & $\sigma_{\psi}<5 \mathrm{deg}$ \\
\hline \hline
\end{tabular}

\section{E. Flight Condition}

Only one flight condition was modeled for the development of this tool and was used in the initial results presented in Section IV. The cruise flight condition was implemented in the development of the tool due to the availability of aerodynamic data given by Napolitano. ${ }^{30}$ This aerodynamic data was used to verify the derived equations of motion by matching the roots of the state matrix to the roots of the transfer function characteristic equation derived in Ref. 30. Additionally, data on the flight condition such as altitude, Mach number, true airspeed, steady-state angle of attack, and dynamic pressure were given. Finally, the mass properties were given in Napolitano, a key component to accurately evaluating the dynamics of any aircraft. Data taken from Napolitano are summarized in Table 3.

The cruise condition was chosen only for the development of the tool. It is well acknowledged that this flight condition is not a key flight condition for sizing of the stabilizers and other flight conditions must be 
added to increase the validity of the analysis.

Table 3. Cessna 182 cruise flight condition. ${ }^{30}$

\begin{tabular}{ccccccccc}
\hline \hline $\begin{array}{c}\text { Altitude } \\
(f t)\end{array}$ & $\begin{array}{c}\overline{\mathrm{U}} \\
(f t / s)\end{array}$ & $\begin{array}{c}\mathrm{M} \\
(-)\end{array}$ & $\begin{array}{c}\text { Dynamic Pressure } \\
\left(l b / f t^{2}\right)\end{array}$ & $\begin{array}{c}\bar{\alpha} \\
(d e g)\end{array}$ & $\begin{array}{c}\mathrm{m} \\
(l b)\end{array}$ & $\begin{array}{c}\mathrm{I}_{\mathrm{X}} \\
\left(s l-f t^{2}\right)\end{array}$ & $\begin{array}{c}\mathrm{I}_{\mathrm{Y}} \\
\left(s l-f t^{2}\right)\end{array}$ & $\begin{array}{c}\mathrm{I}_{\mathrm{Z}} \\
\left(s l-f t^{2}\right)\end{array}$ \\
\hline 5,000 & 220.1 & 0.201 & 49.6 & 0 & 2,650 & 948 & 1,346 & 1,967 \\
\hline \hline
\end{tabular}

\section{Analysis}

\section{A. Aerodynamic Modeling}

Athena Vortex Lattice (AVL), ${ }^{22}$ a code maintained by Mark Drela at the Massachusetts Institute of Technology (MIT), was used to evaluate the aerodynamics of each configuration. This tool was chosen due to the ease of availability, previous user experience, ability to calculate stability derivatives while trimmed in both lateral and longitudinal directions simultaneously, and the low computational cost. The stability derivatives were taken directly from the AVL output and input into the equations of motion described in Section II.

The Cessna 182T Skylane geometry was modeled using 3-view sketches from Refs. 31 and 32. Only the lifting surfaces were modeled in AVL while the non-lifting surfaces were neglected to maintain simplicity of the geometry. The control surfaces were accurately modeled and then modified so the control surfaces were defined as a constant percent chord. This reduces the number of geometry parameters in the tool while the effects on the analysis were insignificant.

Figure 3 shows the Cessna Skylane model pictured in AVL's graphical user interface (GUI) with the non-lifting bodies removed. The center of gravity (CG) position was placed 8.851 feet measured from the propeller spinner as shown in the Pilot's Operating Handbook ${ }^{31}$ and given by Napolitano. ${ }^{30}$

\section{B. Modification of the Geometry}

In this initial study, the only geometrical parameters varied were the horizontal and vertical tail volume coefficients. The vertical tail strake, shown in Fig. 3, was neglected to maintain the trapezoidal geometry parameters. To reduce the number of variables, the horizontal and vertical tail moment arms, aspect ratios, quarter-chord sweeps, dihedral angles, taper ratios, and airfoils were unchanged from the baseline model. Regardless of the change in volume coefficient, the root quarter-chord coordinate locations were identical. Effectively, changing the volume coefficient with these restrictions results only in a change of reference area and span.

The horizontal tail volume coefficient is defined as

$$
C_{H}=\frac{S_{H} l_{H}}{S c}
$$

Traditionally, $l_{H}$ is defined as the length between the wing aerodynamic center and the stabilizer aerodynamic center. For simplicity due to the current fixed CG position in this analysis, the length $l_{H}$ used in Eq. 29 was measured from the CG position to the root quarter-chord point on the stabilizer. Of note, the volume coefficient for the vertical stabilizer is the same equation as Eq. 29 with the subscript $H$ replaced by subscript $V$ and mean aerodynamic chord, $c$, replaced by the span, $b$.

The tail volume coefficients were varied from the Cessna 182 model values of $C_{H}=0.7$ and $C_{V}=0.28$ to $25 \%$ of the baseline over ten equally spaced steps. This resulted in 100 geometries evaluated by the tool, 
each with differing stabilizer areas and spans, taking nearly 50 minutes of computation time. As the tool was still in development, computation time was sacrificed to provide extra data to the user, ensuring it was performing as expected.

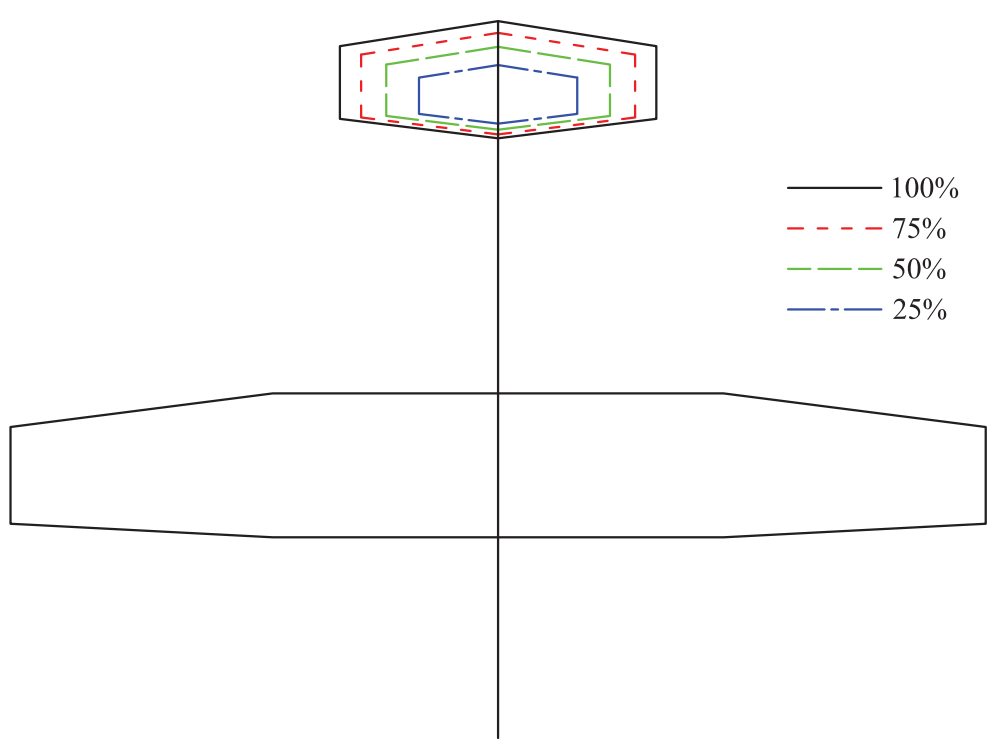

Figure 4. Cessna 182 geometry with modified horizontal tail as percentage of baseline volume coefficient.
Figure 4 shows a drawing from the top view of the AVL geometry with varying horizontal tail volume coefficients. The coefficients shown are 0.70 , $0.52,0.35$, and 0.17 which correspond to $100 \%, 75 \%, 50 \%$, and $25 \%$ of the baseline. The centerline of the fuselage is indicated by the solid line drawn through the symmetric plane.

Any experienced aircraft designer would notice the small horizontal tail area for the $25 \%$ baseline volume coefficient and doubt its ability to achieve adequate handling qualities, much less trim at takeoff conditions. As discussed later in Section IV, all cases passed the dynamic performance checks for adequate handling qualities. However, focusing only on the cruise flight condition is inadequate for accurately sizing the stabilizers, and more constraining flight conditions must be added to the analysis.

\section{Drag Buildup}

To capture all of the drag benefit of reducing the stabilizer area, and including active control in the design process, the reduction in parasitic drag must be calculated from the reduced wetted area. A zero-lift drag coefficient in the cruise condition was given by Napolitano as $C_{D_{0}}=0.027$. This is the total parasitic drag for the Cessna 182 Skylane which was modeled using AVL. Reducing the horizontal and vertical stabilizer areas will reduce the total parasitic skin-friction drag. To model this, the contribution of the baseline empennage to the total parasitic drag was estimated using Raymer's component buildup method where each component is denoted by the subscript $c{ }^{3}$ The subsonic parasitic drag coefficient can be approximated by

$$
\left(C_{D_{0}}\right)_{\text {subsonic }}=\frac{\sum C_{f_{c}} F F_{c} Q_{c} S_{w_{e} t_{c}}}{S}
$$

where $C_{f}$ is the skin-friction drag coefficient, $F F$ is a form factor that estimates the pressure drag due to viscous separation, $Q$ is an interference factor, and $S_{w e t}$ is the estimated wetted area of the component. The miscellaneous, leakage, and protuberance drag terms were ignored. The empennage contribution to $C_{D_{0}}$ was subtracted from the baseline configuration $C_{D_{0}}$ of 0.027 leaving only the wing-body parasitic drag. This allows the stabilizer areas to be varied and the effect on the parasitic drag to be captured. Hence, reduced stabilizer areas result in reduced parasitic drag.

\section{Results and Discussion}

Figure 5 shows the total drag coefficient as a function of the horizontal and vertical tail volume coefficients. As expected, the total drag decreased as the tail volume coefficients were reduced; the total drag was sensitive to both volume coefficients, but had a greater sensitivity to the vertical tail volume coefficient. Figure 6(a) shows the reduction in drag that resulted from decreasing the static margin. The drag benefit seen in Figs. 5 and 6(a) comes from the reduction in wetted area and the resulting decrease in parasitic drag. When trimmed, induced drag for this geometry actually increased with the reduction of static margin as shown in Fig. 6(b). This explains why the total drag was more sensitive to varying the vertical tail volume coefficient as mentioned previously. The resulting increase in induced drag comes not from the reduction of static 
margin but the reduction in the span, and increase in lift coefficient, of the horizontal stabilizer.

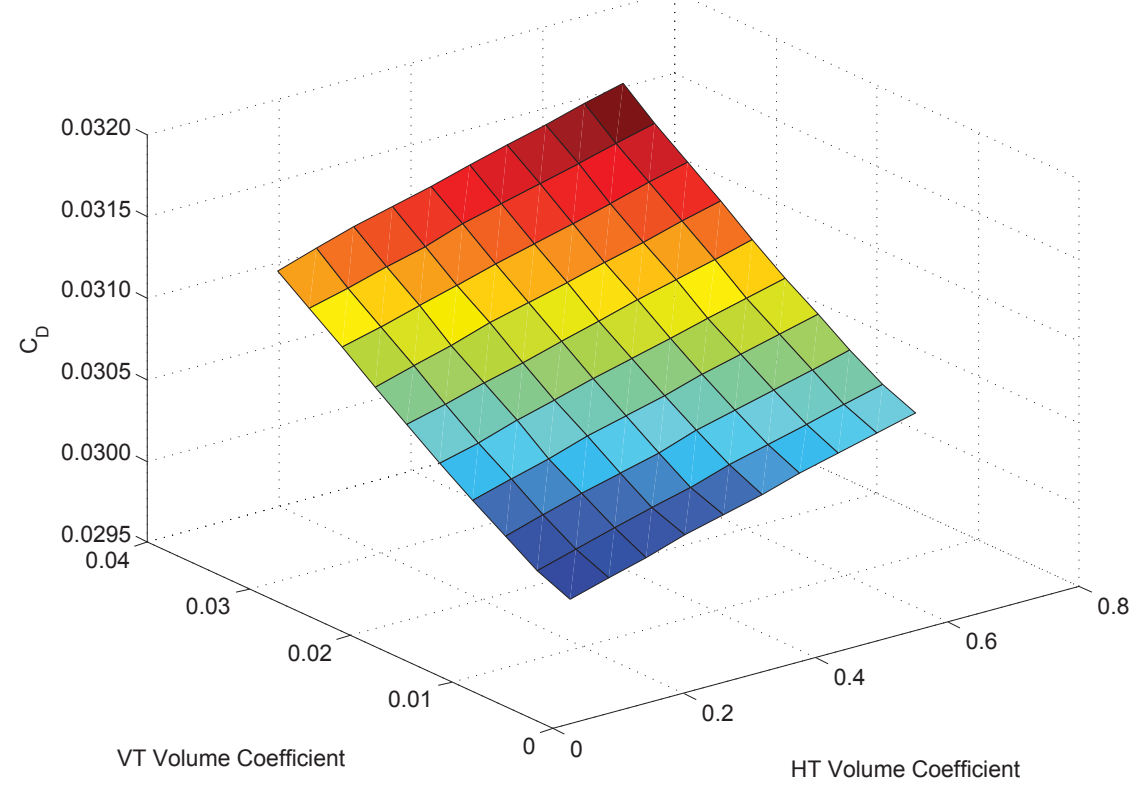

Figure 5. Drag coefficient for varying tail volume coefficients.

The elevator deflection angle, shown in Fig. 7, increased in magnitude as the static margin was decreased. The Cessna 182 baseline geometry required zero elevator deflection to trim at the cruise condition which means the downwash from the main wing provided sufficient induced angle on the horizontal stabilizer to trim. Because the location of the wing and center of gravity were fixed, the horizontal tail has to generate the same amount of moment to trim as the baseline case. As the horizontal tail area and span were decreased, the down lift on the stabilizer decreased requiring the elevator to deflect, increasing the magnitude of the horizontal tail lift coefficient, to achieve trim. As a result, a net increase in configuration induced drag was realized. This indicates that simply reducing the static margin does not decrease induced drag but that a system level design, with static margin being allowed to decrease, must be performed in order to achieve the induced drag benefits of relaxed static stability.

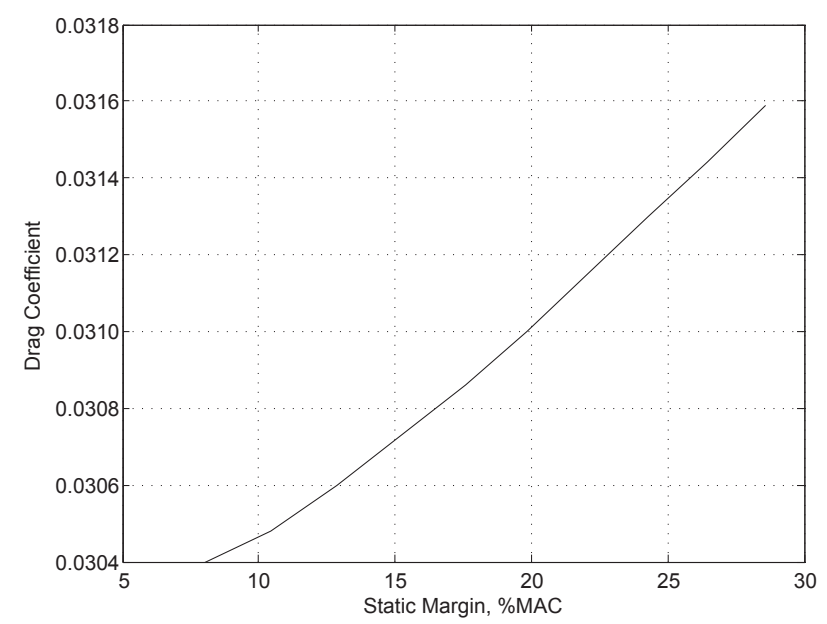

(a) Drag coefficient vs. static margin.

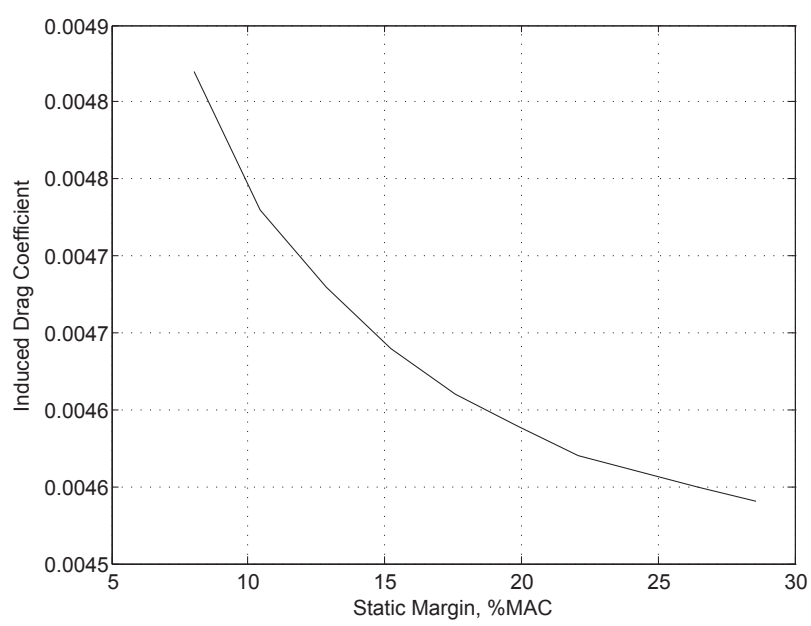

(b) Induced drag coefficient vs. static margin.

Figure 6. Sensitivity of total and induced drag coefficients to static margin.

The accuracy of the aerodynamic model should also be considered when designing for relaxed static stability. AVL predicted a baseline static margin of nearly $30 \%$ which was much greater than the actual static margin. Both the engine and fuselage, neglected in the AVL model, will shift the neutral point forward 
decreasing the static margin. This modeling error causes the geometry to be overly stable in the analysis of the dynamic response.

Initially, using moderate atmospheric disturbances with a probability of exeedance level of $10^{-3}$, none of the configurations passed the heading hold turbulence check specified in SAE-AS94900. This resulted from the active control system not sufficiently driving the rudder in continuous turbulence. A great feature of the LQR is the flexibility in allowing for adjustments in the weighting matrices if a desired performance is not achieved. Due to the consistent failures in the heading hold check, the $\psi$ weighting term in Eq. 14 was increased by a factor of 10 , placing heavier penalties on non-zero values of yaw. This simple adjustment enabled all configurations to achieve adequate dynamic performance in the cruise flight condition. It should be noted, again, that the cruise flight condition alone does not adequately stress the control system for sizing the stabilizer surfaces. Additional flight conditions must be added to ensure proper sizing of the stabilizer surfaces.

Figures $8-12$ show the baseline configuration's dynamic response with the heavier yaw weighting to perturbations, continuous gust fields, and discrete gusts. These disturbances were used to check the dynamic performance of the active control system as summarized in Table 2. The airspeed hold and longitudinal gust responses were omitted for brevity due to their similarity to the pitch hold and vertical gust responses.

Time histories of the transient response to the perturbations specified by SAE-AS94900 are shown in Figs. 8 and 9 . The figures were separated into longitudinal and lateral states, showing both the short time response-less than ten seconds - and the long time response - total response over $100 \mathrm{sec}-$ onds. All control surface states shown in Figs. 8, 9, 11, and 12 are the total

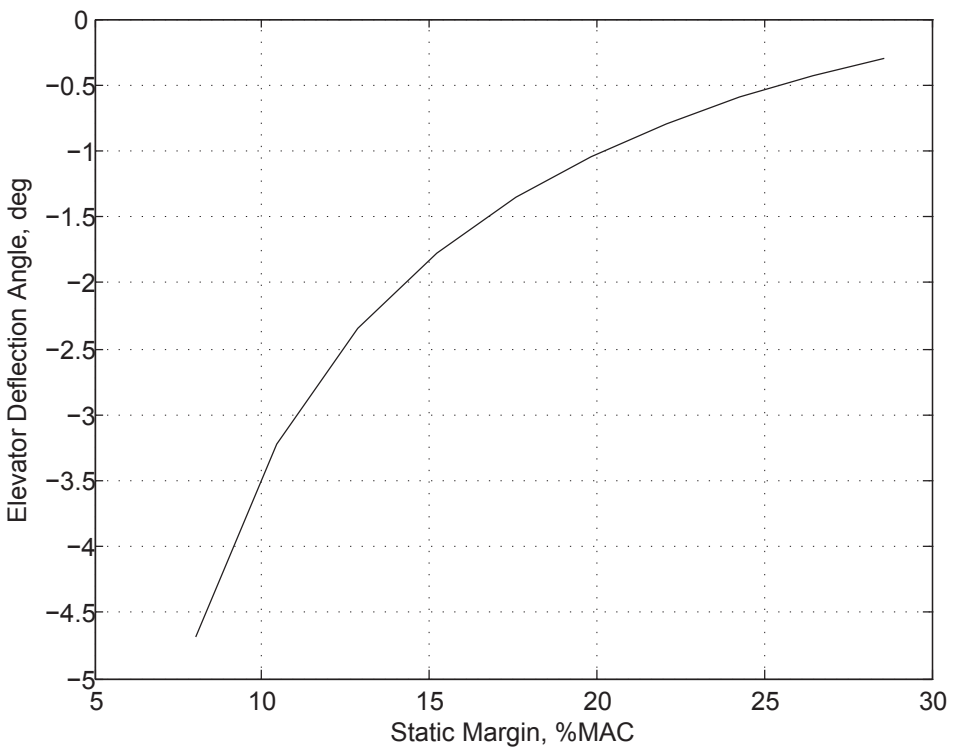

Figure 7. Elevator deflection angle versus static margin. deflection angles, the sum of the perturbation and the steady-state deflection angle. Clearly visible in the open-loop response of Fig. 8 is the phugoid mode which has clearly been attenuated with little control power. Figure 9 shows the unstable spiral mode in the open-loop response that has been stabilized through a combination of aileron and rudder control surface deflections.

Power spectral densities of the mean-squared response are plotted in Fig. 10. With a natural frequency of $0.19 \mathrm{rad} / \mathrm{s}$, the phugoid sensitivity can clearly be seen in the vertical continuous turbulence responses as a spike in the mean-squared response of the open-loop system. Less obvious is the short period mode, which had an open-loop natural frequency of $7.66 \mathrm{rad} / \mathrm{s}$, that can be seen as a slight hump in the angle of attack, pitch rate, and pitch angle. The spike in the response of the lateral states of Fig. 10 correspond to the dutch roll natural frequency of $5.14 \mathrm{rad} / \mathrm{s}$. The active control system successfully reduced the magnitude of the response of the phugoid and dutch roll modes while the short period response in turbulence was unchanged.

Figures 11 and 12 show the response to a lateral and vertical discrete gust. As with the pitch perturbations, the phugoid is quite visible in the open-loop response. Due to the model being extremely stable at cruise, even in a discrete gust, the required elevator deflection of the closed-loop system was extremely small while providing a heavily damped response. The closed-loop lateral gust response, Fig. 11, provides increased damping when compared to the open-loop response, as would be expected. Heavier weighting in the performance index on the heading angle, $\psi$, required to meet the required heading hold performance results in a sacrifice in the roll angle, obtaining improved heading angle response. The heading perturbation state of Fig. 11 deviates from steady-state in response to the gust but immediately returns to the steady-state value, whereas the roll perturbation state has two full oscillation cycles before returning to the steady state. 

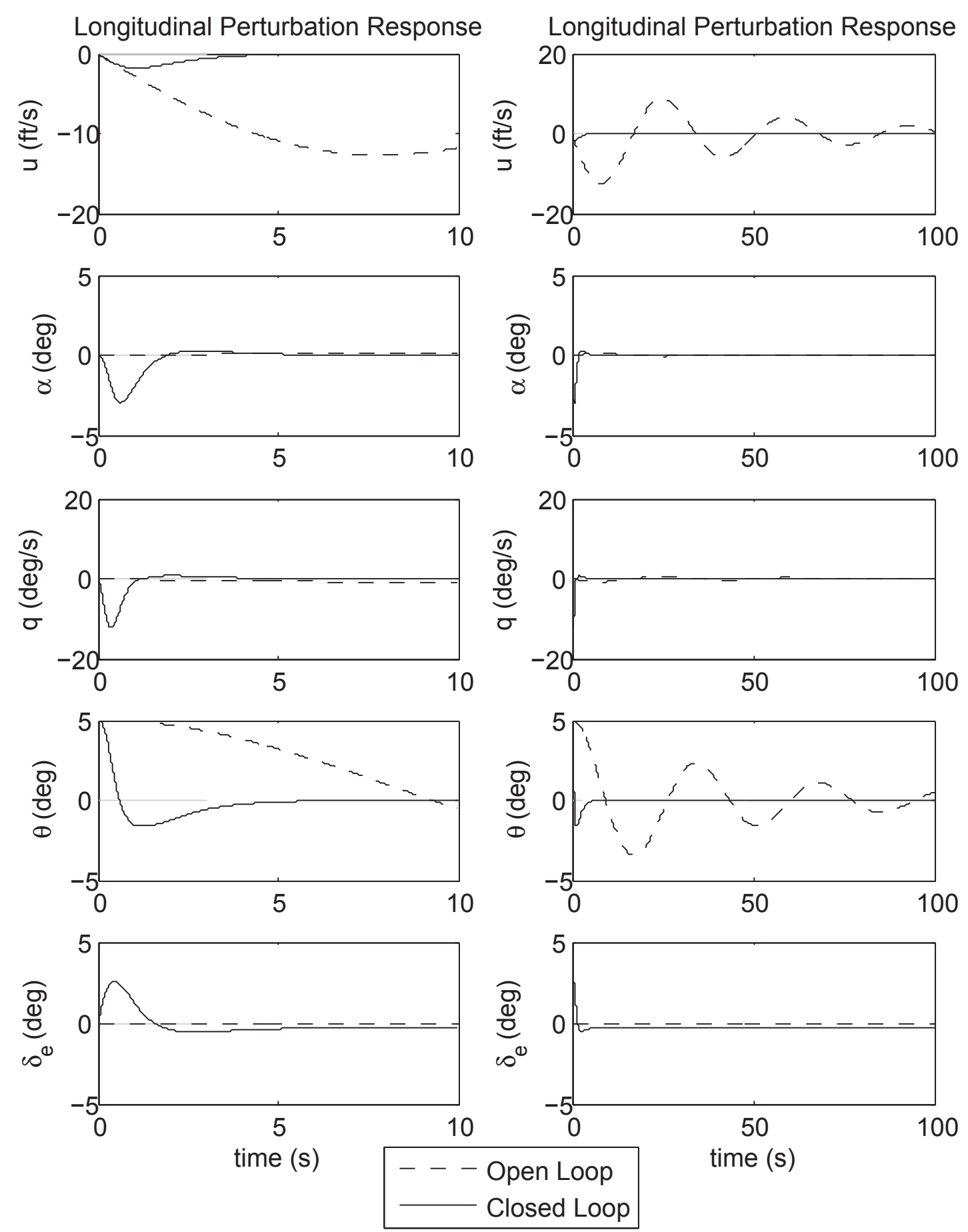

Figure 8. Pitch hold perturbation check. 

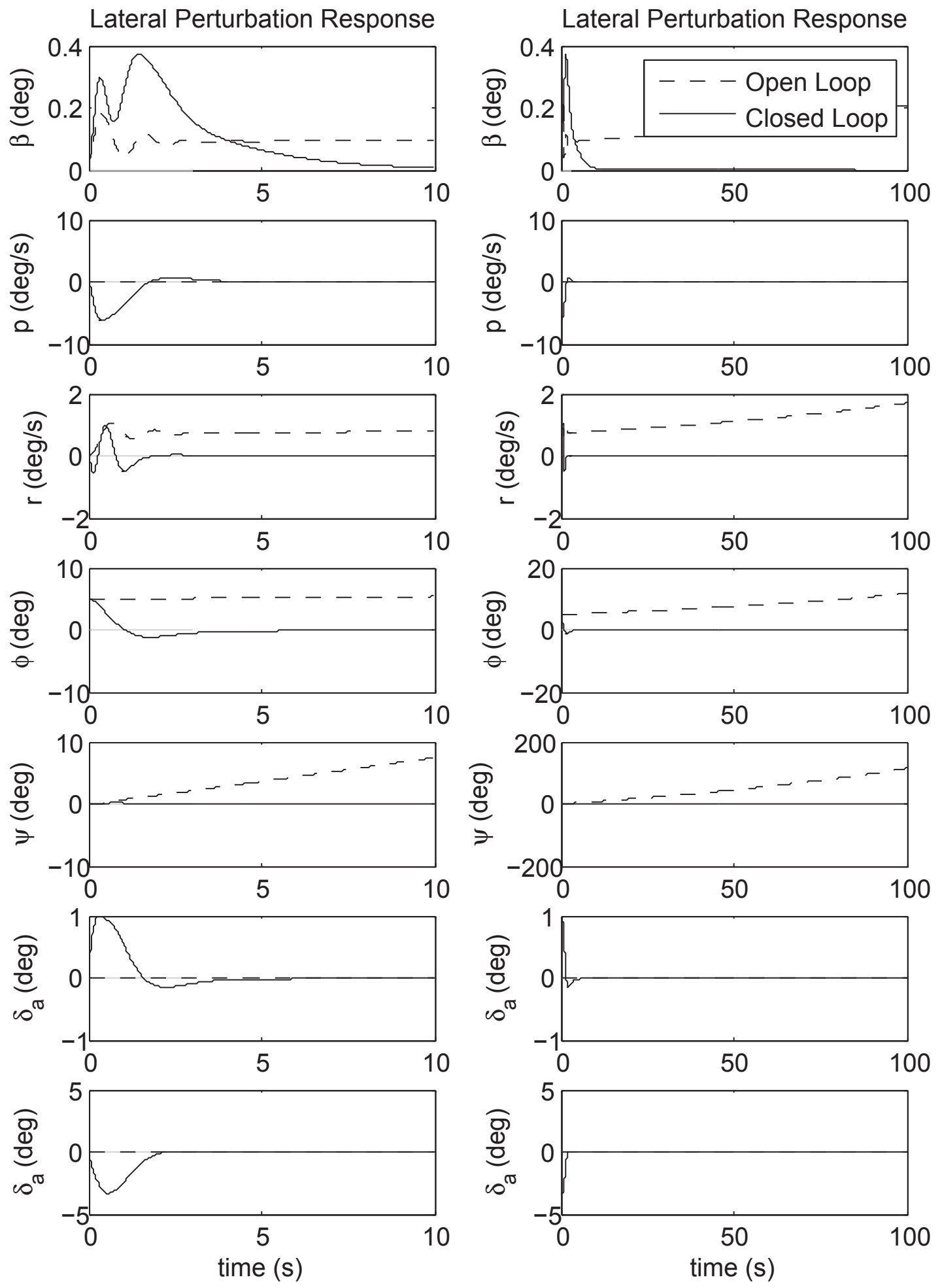

Figure 9. Roll hold perturbation check. 

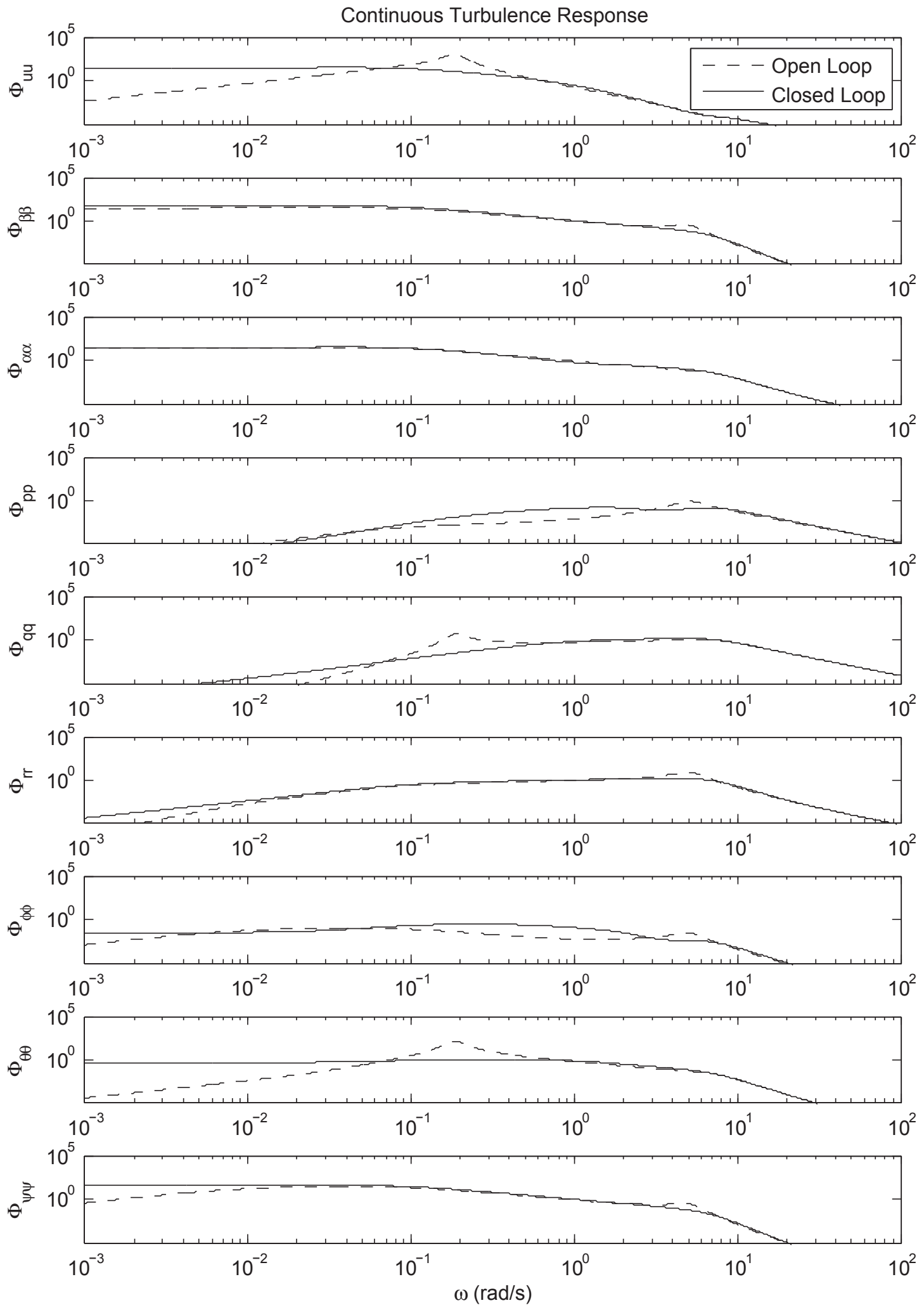

Figure 10. Continuous turbulence response. 

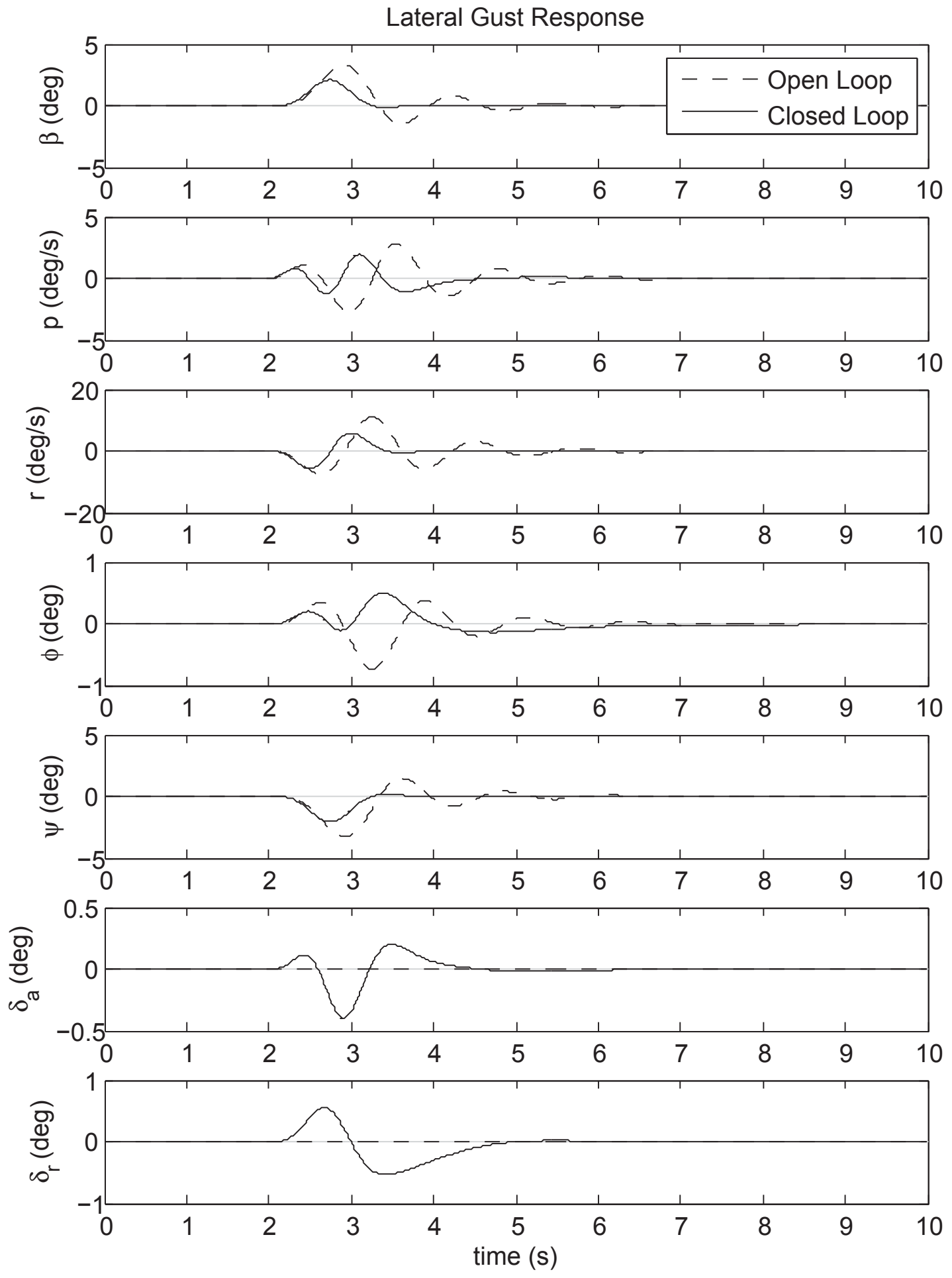

Figure 11. Lateral discrete gust response. 

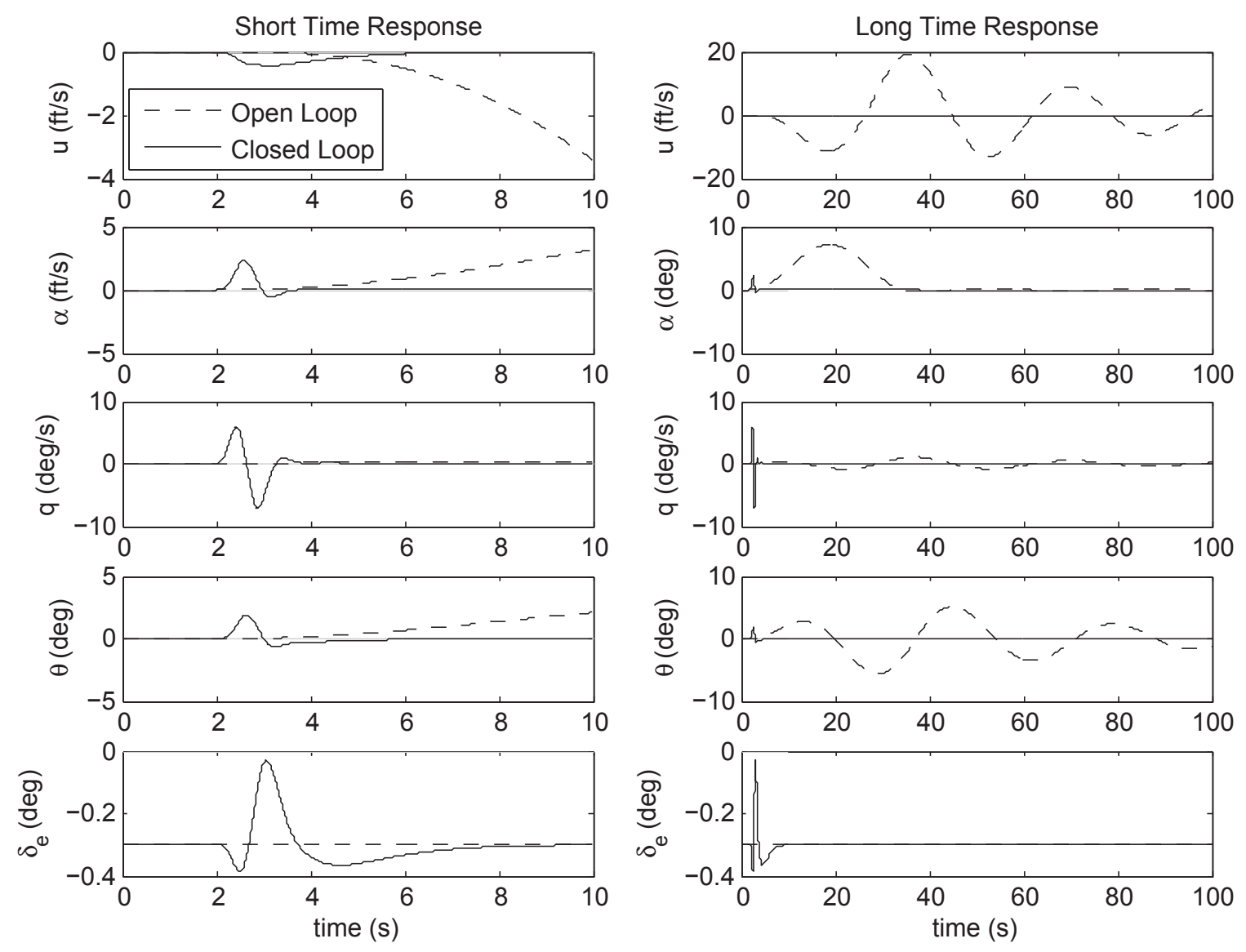

Figure 12. Vertical discrete gust response.

\section{Conclusions}

A multidisciplinary optimization, conceptual design tool methodology was presented along with initial results. An LQR controller was developed along with a methodology for selecting the weighting matrices that reduces number of elements that must be chosen. Using control system performance requirements from SAE-AS94900, dynamic constraints were implemented and tested using perturbation simulations, continuous atmospheric turbulence modeling, and discrete gust simulations. The geometry was varied and active control used to stabilize the system to allow for a reduction in wetted area and static stability. Initial results showed that a heavier weighting on the heading state was required to meet the continuous turbulence performance requirements of SAE-AS94900. After the adjustment in heading state weighting, drag reductions were achievable through the reduction of the horizontal and vertical stabilizers while maintaining acceptable dynamic response. By implementing active control using a linear quadratic regulator, with a simplified structure to reduce weighting matrix decisions, the design process, including active control, has been shown to be feasible. As indicated in the results, a decrease in static margin does not guarantee a reduction in induced drag; to realize the full benefits of relaxed static stability, the entire configuration needs to be given the freedom to vary geometric parameters without the traditional restriction on minimum static margin.

Continued expansion of the tool is necessary to incorporate all the traditional design disciplines to make it fully functional. The results indicate the importance of identifying the constraining flight conditions when sizing the stabilizers as the cruise flight condition did not heavily load the active control system.

\section{Future Work}

Additional flight conditions will be added as the cruise condition used here was not a constraining flight condition for sizing control effectors. The atmospheric disturbances model will also be enhanced to include 
the induced rotation rates resulting from each surface entering the disturbance at slightly different times. This effect will be large for aircraft with large separations between the main wing and horizontal stabilizer. The discrete gust magnitude was calculated using Ref. 28 and had a probability of exceedance of $10^{-2}$, a light disturbance level. Greater disturbance levels will be added to the normalized discrete gust calculation to account for more severe gust levels. These additions will increase the validity of the atmospheric disturbance modeling and place a greater workload on the active control system.

The work presented herein gave an indication of the intentions of this multidisciplinary design tool. A sizing and weights estimation tool needs to be added to allow for the variation of the entire geometry in the design space. The Flight Optimization Software (FLOPS) code will be attached to the method discussed and will perform the sizing, weight estimates, performance estimates, and parasitic drag buildup. A baseline FLOPS model of an advanced, 737-800 class, double-bubble fuselage configuration called the D8 will be used. A global optimizer will then be allowed to vary numerous geometric parameters with the goal of including flight dynamics and control into the conceptual design process, helping to achieve the Aeronautics Research Mission Directorate goal of reduced fuel burn.

\section{Appendix}

The matrices of the fully coupled, perturbation equations are provided below. These matrices are used in Eqs. 1, 3, and 6.

$$
E=\left[\begin{array}{ccccccccc}
m & 0 & \frac{C_{D_{\dot{\alpha}}} S c q_{\infty}}{2 \bar{U}^{2}} & 0 & 0 & 0 & 0 & 0 & 0 \\
0 & m-\frac{C_{Y_{\dot{\beta}}} S b q_{\infty}}{2 \bar{U}^{2}} & 0 & 0 & 0 & 0 & 0 & 0 & 0 \\
0 & 0 & m+\frac{C_{L_{\dot{\alpha}}} S c q_{\infty}}{2 \bar{U}^{2}} & 0 & 0 & 0 & 0 & 0 & 0 \\
0 & -\frac{C_{l_{\dot{\beta}}} S b^{2} q_{\infty}}{2 \bar{U}^{2}} & 0 & I_{X}^{\prime} & 0 & -I_{X Z}^{\prime} & 0 & 0 & 0 \\
0 & 0 & -\frac{C_{m_{\dot{\alpha}}} S c^{2} q_{\infty}}{2 \bar{U}^{2}} & 0 & I_{Y}^{\prime} & 0 & 0 & 0 & 0 \\
0 & -\frac{C_{n_{\dot{\beta}}} S b^{2} q_{\infty}}{2 \bar{U}^{2}} & 0 & -I_{X Z}^{\prime} & 0 & I_{Z}^{\prime} & 0 & 0 & 0 \\
0 & 0 & 0 & 0 & 0 & 0 & 1 & 0 & -\sin (\bar{\theta}) \\
0 & 0 & 0 & 0 & 0 & 0 & 0 & \cos (\bar{\phi}) & \cos (\bar{\theta}) \sin (\bar{\phi}) \\
0 & 0 & 0 & 0 & 0 & 0 & 0 & -\sin (\bar{\phi}) & \cos (\bar{\phi}) \cos (\bar{\theta})
\end{array}\right]
$$

$\hat{A}(:, 1: 3)=\frac{S q_{\infty}}{\bar{U}} \times$

$\left[\begin{array}{ccc}C_{T_{X_{u}}}-C_{D_{u}}+C_{T_{Z_{u}}} \bar{\alpha}+\frac{2 g m \sin (\bar{\theta})}{S q_{\infty}} & \frac{\bar{R} \bar{U} m}{S q_{\infty}}-C_{D_{\beta}} & \bar{C}_{L}-C_{D_{\alpha}}+C_{T_{X_{\alpha}}}+C_{T_{Z_{\alpha}}} \bar{\alpha} \\ \frac{C_{T_{Y_{u}}} q_{\infty}+C_{Y_{u}} q_{\infty}}{q_{\infty}+\frac{\bar{R} \bar{U} m-2 g m \cos (\bar{\theta}) \sin (\bar{\phi})}{S q_{\infty}}} & C_{T_{Y_{\beta}}}+C_{Y_{\beta}} & 0 \\ C_{T_{Z_{u}}}-C_{L_{u}}-C_{T_{X_{u}}} \bar{\alpha}-\frac{2 g m \cos (\bar{\phi}) \cos (\bar{\theta})}{S q_{\infty}} & 0 & C_{T_{Z_{\alpha}}}-C_{L_{\alpha}}-\bar{C}_{D}-C_{T_{X_{\alpha}}} \bar{\alpha} \\ b\left(C_{T_{l_{u}}}+C_{l_{u}}+C_{T_{n_{u}}} \bar{\alpha}\right) & b\left(C_{T_{l_{\beta}}}+C_{l_{\beta}}+C_{T_{n_{\beta}}} \bar{\alpha}\right) & -\bar{C}_{n} b \\ c\left(C_{T_{m_{u}}}+C_{m_{u}}\right)-\frac{2 I_{X Z}^{\prime} \bar{R}^{2}}{S q_{\infty}} & b\left(C_{T_{n_{\beta}}}+C_{n_{\beta}}-C_{T_{l_{\beta}}} \bar{\alpha}\right) & c\left(C_{T_{m_{\alpha}}}+C_{m_{\alpha}}\right) \\ b\left(C_{T_{n_{u}}}+C_{n_{u}}-C_{T_{l_{u}}} \bar{\alpha}\right) & 0 & \bar{C}_{l} b \\ 0 & 0 & 0 \\ 0 & 0 & 0\end{array}\right]$




$$
\begin{aligned}
& \hat{A}(:, 4: 9)=\frac{S q_{\infty}}{\bar{U}} \times \\
& {\left[\begin{array}{cccccc}
0 & -\frac{C_{D_{q}} c}{2} & 0 & 0 & -\frac{\bar{U} g m \cos (\bar{\theta})}{S q_{\infty}} & 0 \\
\frac{C_{Y_{p}} b}{2} & 0 & \frac{C_{Y_{r}} b}{2}-\frac{\bar{U}^{2} m}{S q_{\infty}} & \frac{\bar{U} g m \cos (\bar{\phi}) \cos (\bar{\theta})}{S q_{\infty}} & -\frac{\bar{U} g m \sin (\bar{\phi}) \sin (\bar{\theta})}{S q_{\infty}} & 0 \\
0 & \frac{\bar{U}^{2} m}{S q_{\infty}-\frac{C_{L_{q}} c}{2}} & 0 & -\frac{\bar{U} g m \cos (\bar{\theta}) \sin (\bar{\phi})}{S q_{\infty}} & -\frac{\bar{U} g m \cos (\bar{\phi}) \sin (\bar{\theta})}{S q_{\infty}} & 0 \\
\frac{C_{l_{p} b^{2}}}{2} & \frac{\bar{R} \bar{U}\left(I_{Y}^{\prime} I_{Z}^{\prime}\right)}{S q_{\infty}} & \frac{C_{l_{r}} b^{2}}{2} & 0 & 0 & 0 \\
\frac{\bar{R} \bar{U}\left(I_{X}^{\prime}-I_{Z}^{\prime}\right)}{S q_{\infty}} & \frac{C_{m_{q}} c^{2}}{2} & \frac{2 I_{X Z}^{\prime} \bar{R} \bar{U}}{S q_{\infty}} & 0 & 0 & 0 \\
\frac{C_{n_{p}} b^{2}}{2} & -\frac{I_{X Z}^{\prime} \bar{R} \bar{U}}{S q_{\infty}} & \frac{C_{n_{r} b^{2}}}{2} & 0 & 0 & 0 \\
\frac{2}{S} & 0 & 0 & 0 & 0 & 0 \\
0 & \frac{\bar{U}}{S q_{\infty}} & 0 & 0 & 0 & 0 \\
0 & 0 & \frac{\bar{U}}{S q_{\infty}} & 0 & 0 & 0
\end{array}\right]} \\
& \hat{B}=S q_{\infty}\left[\begin{array}{ccccccccc}
-C_{D_{\delta_{e}}} & 0 & -C_{L_{\delta_{e}}} & 0 & C_{m_{\delta_{e}}} c & 0 & 0 & 0 & 0 \\
0 & C_{Y_{\delta_{a}}} & 0 & C_{l_{\delta_{a}}} b & 0 & C_{n_{\delta_{a}}} b & 0 & 0 & 0 \\
0 & C_{Y_{\delta_{r}}} & 0 & C_{l_{\delta_{r}}} b & 0 & C_{n_{\delta_{r}}} b & 0 & 0 & 0
\end{array}\right]^{\mathrm{T}}
\end{aligned}
$$

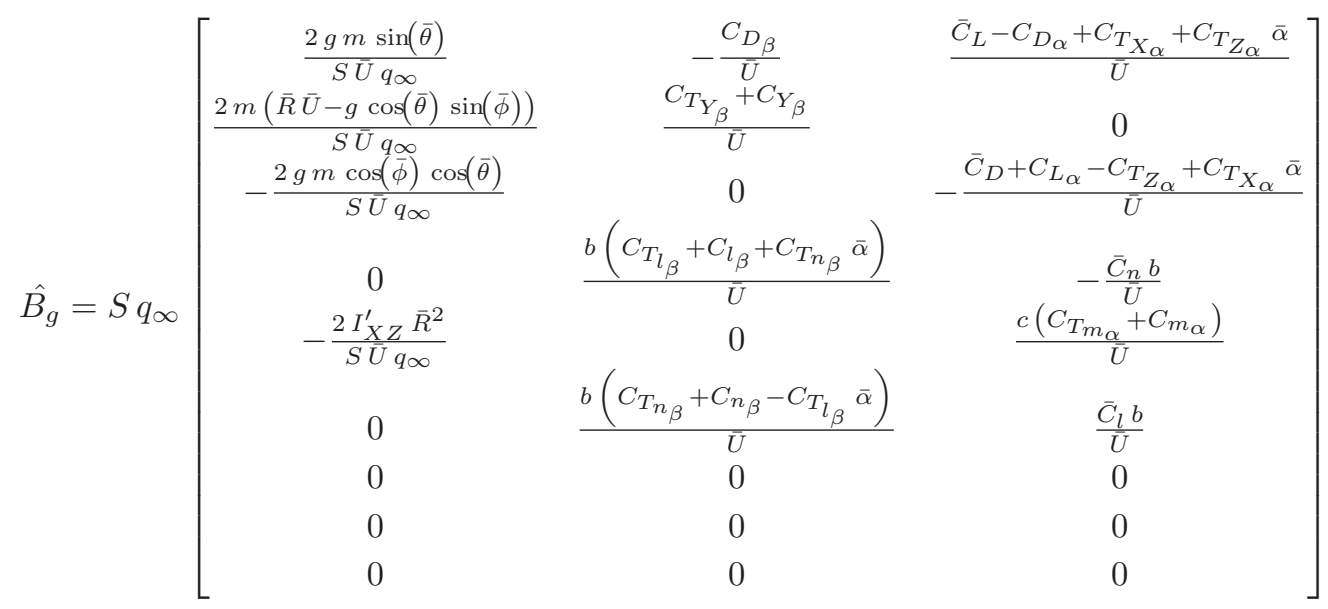

$$
\begin{aligned}
& E_{\text {aug }}=\left[\begin{array}{c:ccc}
E & \multicolumn{3}{c}{0} \\
\hdashline & 1 & 0 & 0 \\
0 & 0 & 1 & 0 \\
& 0 & 0 & 1
\end{array}\right]
\end{aligned}
$$

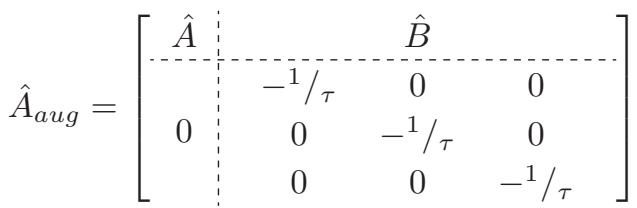

$$
\begin{aligned}
& \hat{B}_{u}=\left[\begin{array}{rrrrrrrrrcrr}
0 & 0 & 0 & 0 & 0 & 0 & 0 & 0 & 0 & 1 / \tau & 0 & 0 \\
0 & 0 & 0 & 0 & 0 & 0 & 0 & 0 & 0 & 0 & 1 / \tau & 0 \\
0 & 0 & 0 & 0 & 0 & 0 & 0 & 0 & 0 & 0 & 0 & 1 / \tau
\end{array}\right]^{\mathrm{T}}
\end{aligned}
$$

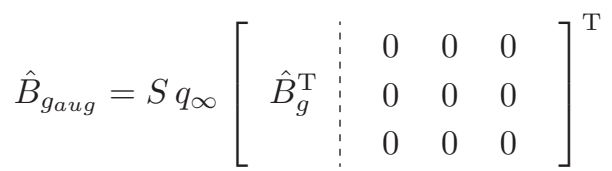


The symbols used in Eqs. 31-39 are defined as

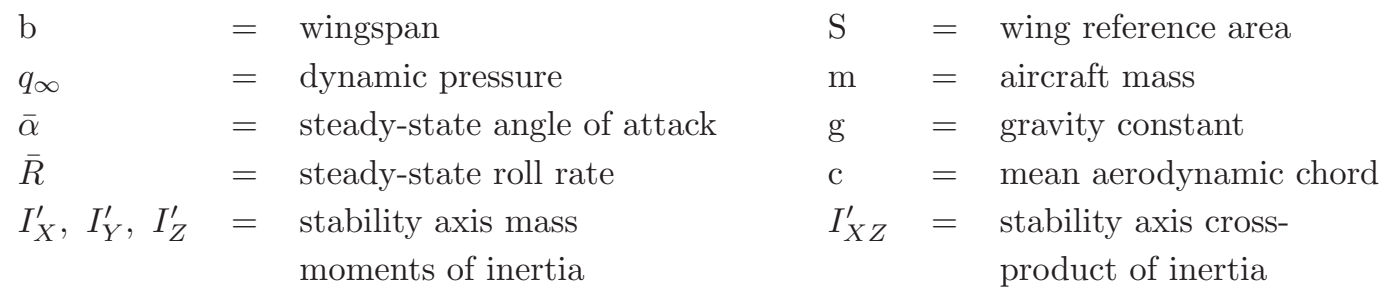

\section{References}

${ }^{1}$ Roskam, J., Flight Dynamics and Automatic Flight Control: Pt. I, DARcorporation, Lawrence, KS, 2011.

2 "The Effect of High Altitude and Center of Gravity on The Handling Characteristics of Swept-wing Commercial Airplanes," http://www.boeing.com/commercial/aeromagazine/aero_02/textonly/fo01txt . html [retrieved November 2013].

${ }^{3}$ Raymer, D. P., Aircraft Design: A Conceptual Approach, 4th ed., AIAA Education Series, American Institute of Aeronautics and Astronautics, Reston, VA, 2006.

${ }^{4}$ Roskam, J., Airplane Design, Pt. II: Preliminary Configuration Design and Integration of the Propulsion System, DARcorporation, Ottawa, KS, 1997.

${ }^{5}$ Nicolai, L. M. and Carichner, G. E., Fundamentals of Aircraft and Airship Design: Volume I, AIAA Education Series, American Institute of Aeronautics and Astronautics, Reston, VA, 2010.

${ }^{6}$ Stevens, B. L. and Lewis, F. L., Aircraft Control and Simulation, 2nd ed., John Wiley \& Sons, Inc., Hoboken, NJ, 2003.

${ }^{7}$ Pamadi, B. N., Performance, Stability, Dynamics, and Control of Airplanes, 2nd ed., AIAA Education Series, American Institute of Aeronautics and Astronautics, Reston, VA, 2004.

${ }^{8}$ Coleman, G. and Chudoba, B., "A Generic Stability and Control Tool for Conceptual Design: Prototype System Overview," AIAA Aerospace Sciences Meeting and Exhibit, Reno, NV, 2007, AIAA-2007-659.

${ }^{9}$ Perez, R. E., Liu, H. H. T., and Behdinan, K., "Multidisciplinary Optimization Framework for ControlConfiguration Integration in Aircraft Conceptual Design," Journal of Aircraft, Vol. 43, No. 6, 2006, pp. 1937-1947.

${ }^{10}$ Perez, R. E., Liu, H. H. T., and Behdinan, K., "Flight Dynamics and Control Multidisciplinary Integration in Aircraft Conceptual Design Optimization," AIAA/ISSMO Multidisciplinary Analysis and Optimization Conference, American Institute of Aeronautics and Astronautics, Albany, NY, 2004, AIAA-2004-4435.

${ }^{11}$ Perez, R. E., Liu, H. H. T., and Behdinan, K., "Relaxed Static Stability Aircraft Design via Longitudinal Control-Configured Multidisciplinary Design Optimization Methodology," Canadian Aeronautics and Space Journal, Vol. 52, No. 1, 2006, pp. 1-14.

${ }^{12}$ Chudoba, B. and Smith, H., "A Generic Stability and Control Methodology for Novel Aircraft Conceptual Design," AIAA Atmospheric Flight Mechanics Conference and Exhibit, Austin, TX, 2003, AIAA-20035388.

${ }^{13}$ Cabral, L. V. and Paglione, P., "Conceptual Design of Families of Aircraft Using Multi Objective Design Optimization Theory and Genetic Algorithm Techniques," 6th World Congresses of Structural and Multidisciplinary Optimization, Rio de Janeiro, 2005.

${ }^{14}$ Morris, C. C., Sultan, C., Allison, D. L., Schetz, J. A., and Kapania, R. K., "Towards Flying Qualities Constraints in the Multidisciplinary Design Optimization of a Supersonic Tailless Aircraft," AIAA Aviation Technology, Integration, and Operations Conference, Indianapolis, IN, 2012, AIAA-2012-5517. 
${ }^{15}$ Boyd, S., Ghaoui, L. E., Feron, E., and Balakrishnan, V., Linear Matrix Inequalities in System and Control Theory, Vol. 15 of Studies in Applied and Numerical Mathematics, Society for Industrial and Applied Mathematics (SIAM), 1994.

${ }^{16}$ Kaminer, I. I., Howard, R. M., and Buttrill, C. S., "Development of Closed-Loop Tail-Sizing Criteria for a High Speed Civil Transport," Journal of Aircraft, Vol. 34, No. 5, 1997.

${ }^{17}$ Morris, C. C., Sultan, C., Schetz, J. A., and Kapania, R. K., "Variance Constrained Flying Qualities Metrics for Conceptual Design Feasibility Studies," AIAA Guidance, Navigation, and Control Conference, Boston, MA, 2013, AIAA-2013-4927.

${ }^{18}$ Richardson, T. S., Beaverstock, C., Isikveren, A., Meheri, A., Badcock, K., and Ronch, A. D., "Analysis of the Boeing 747-100 using CEASIOM," Progress in Aerospace Sciences, Vol. 47, 2011, pp. 660-673.

${ }^{19}$ Rizzi, A., Eliasson, P., McFarlane, C., Goetzendorf-Grabowski, T., and Vos, J., "Virtual-Aircraft Design \& Control of TransCruiser - a Canard Configuration," AIAA Atmospheric Flight Mechanics Conference, Toronto, Ontario, Canada, 2010, AIAA-2010-8245.

${ }^{20}$ von Kaenel, R., Oppelstrup, J., Goetzendorf-Grabowski, T., Ghoreyshi, M., Cavagna, L., and Bérard, A., "CEASIOM: Simulating Stability \& Control with CFD/CSM in Aircraft Conceptual Design," International Congress of the Aeronautical Sciences, Anchorage, AK, 2008.

${ }^{21}$ Rizzi, A., "Modeling and Simulating Aircraft Stability \& Control - the SimSAC Project," AIAA Atmospheric Flight Mechanics Conference, Toronto, Ontario, Canada, 2010, AIAA-2010-8238.

${ }^{22}$ Drela, M., "Athena Vortex Lattice Manual," 2010, http://web.mit.edu/drela/Public/web/avl/av1_ doc.txt [retrieved January 2013].

${ }^{23}$ SAE International, Aerospace - Flight Control Systems - Design, Installation, and Test of Piloted Military Aircraft, General Specification For, July 2007, SAE-AS94900.

${ }^{24}$ Etkin, B. and Reid, L. D., Dynamics of Flight, John Wiley \& Sons, Inc., Hoboken, NJ, 3rd ed., 1996, pp. $132-133$.

${ }^{25}$ Kalman, R. E., "On the General Theory of Control Systems," Automatic and Remote Control (Proc. IFAC Moscow 1960), Vol. I, Butterworth, London, 1961, pp. 481-492.

${ }^{26}$ Levine, W. and Athans, M., "On the Determination of the Optimal Constant Output Feedback Gains for Linear Multivariable Systems," IEEE Transactions on Automatic Control, Vol. AC-15, No. 1, February 1970, pp. 44-48.

${ }^{27}$ Department of Defense, Flying Qualities of Piloted Aircraft, January 1990, MIL-STD-1797A.

${ }^{28}$ Roskam, J., Flight Dynamics of Rigid and Elastic Airplanes: Pt. II, Jan Roskam, Lawrence, KS, 1972.

${ }^{29}$ MIL-F-8785C, "U.S. Dept. of Defense Military Specification: Flying Qualities of Piloted Airplanes," Tech. rep., November 1980.

30 Napolitano, M. R., Aircraft Dynamics: From Modeling to Simulation, John Wiley \& Sons, Inc., Hoboken, NJ, 2012.

${ }^{31}$ Cessna Aircraft Company, Wichita, KS, Pilot's Operating Handbook and FAA Approved Airplane Flight Manual: Skylane, December 2007.

${ }^{32}$ Cessna Aircraft Company, Wichita, KS, Specification and Description: Exhibit "A", January 2012. 